\section{Comment on}

\section{"Initial-recall grouping in free-recall learning," by John A. Robinson}

\author{
ISABEL M. BIRNBAUM \\ University of California. Irvine, Calif. 92664
}

Robinson (1970) inferred that the maintenance of organization of initially recalled words retards free-recall learning (FRL). It is argued here that his data do not permit this inference.

Robinson was interested in "the extent 10 which words recalled on the first trial were grouped together in subsequent recall periods" in a standard FRL task. The question that he raised was "whether the amount or consistency of such initial-recall grouping (IRG) was systematically related to level of performance."

In Robinson's experiment, 21 Ss learned a 10 -word list for 10 study-test trials. The Ss were then subdivided into three groups on the basis of a measure of initial-recall grouping, $P_{11}$. On a given recall trial, $P_{11}$ is the ratio of the number of adjacent pairs of initially recalled words to the maximum possible number of such pairs. Robinson pointed out that the value of $P_{11}$ is independent of the number of "old" (i.e., initially recalled) words present on a given trial. He failed to note, however, that the expected value of $P_{11}$ is not independent of the ratio of "old" to "new" items (Birnbaum, 1968). Consider, for example,
Ss who recall a total of five old items on a particular trial. Those Ss who recall, in addition, only one new item have a relatively high expected value of $P_{1_{1}}$. That is, any arrangement of one new and five old items will produce a high number of old items adjacent to each other. The range of values of $P_{11}$ in this case is .75-1.00. On the other hand, an $S$ who recalls five old and four new items can score between 0.00 and 1.00 on $P_{11}$. If $S$ is a slow learner and adds relatively few new words after the first trial of FRL, his score on $P_{11}$ must be high simply as a consequence of the high ratio of old to new items recalled.

Robinson (1970) calculated $P_{11}$ for each $S$ on each recall trial (Trials 2-10). The Ss were rank ordered on the basis of average $P_{11}$ over all trials; they were then subdivided into three groups comprising Ss with high $(\mathrm{H})$, medium $(\mathrm{M})$, and low $(\mathrm{L})$ average $P_{11}$ scores. The major finding of the study was that the $\mathrm{H}, \mathrm{M}$, and $\mathrm{L}$ groups differed significantly in rate of FRL. Degree of $P_{11}$ was negatively related to overall performance in FRL.

The question of concern is whether or not high initial-recall grouping (high $P_{11}$ ) is causally related to rate of learning, as Robinson concluded. Selection of Ss on the basis of high, medium, and low $P_{11}$ may simultaneously select for slow, average, and fast learners, respectively. A learner who adds few new items after Trial 1 will inevitably score high on $P_{11}$, even if output order is random. This problem is exaggerated when Ss begin with a high number of words recalled on Trial 1 and the entire list is only 10 words long. A learner who starts low on Trial 1 but adds many new items on subsequent trials can score high on $P_{11}$ but can also score as low as zero. In short, the opportunities for low $P_{11}$ scores differ markedly for slow and fast learners. Since the selection of high, medium, and low "initial-recall organizers" is not independent of an S's rate of FRL, no conclusions can be drawn regarding the effects of initial-recall grouping $\left(P_{11}\right)$ on rate of learning.

\section{REFERENCES}

BIRNBAUM, I. M. Free-recall learning as a function of prior-list organization. Journal of Verbal Learning \& Verbal Behavior, 1968, 7 , 1037-1042.

ROBINSON, J. A. Initial-recall grouping in free-recall learning. Psychonomic Science, $1970,20,67-68$.

\title{
Reply to Birnbaum
}

\author{
JOHN A. ROBINSON \\ University of Louisville, Louisville, Ky. 40208
}

Robinson (1970) reported a negative correlation between average degree of initial-recall grouping (IRG) and overall performance in a free-recall learning task. Birnbaum (1970) has pointed out that the expected value of the grouping measure is not independent of S's rate of learning, i.e., IRG and performance are necessarily correlated, though to an indeterminate degree. The point is well taken, and my attempt to interpret the data in a causal manner is certainly statistically vulnerable. I still believe. however, that internal consistency favors my interpretation.
It is worth emphasizing an aspect of those data that Birnbaum has overlooked. viz, that the composition of recall was quite labile (see Table 2, p. 68 of Robinson, 1970). The fact that $\mathrm{Ss}$ in the $\mathrm{H}$ and $M$ groups rarely reproduced all of the initially recalled words means that the expected value of $P_{11}$ cannot simply be deduced from the total number of words recalled. For example, if $S$ recalls two new and four old words, the range of possible values for $P_{11}$ is from .33 to 1.00 . Thus, Birnbaum's example, while pertinent to her statistical argument, is not characteristic of the data in question. In fact, as was noted in my original discussion, the lability of recall seems to be emerging as one of the most reliable concomitants of organizational conflict.

\section{REFERENCES}

BIRNBAUM, I. M. Comment on "Initial-recall grouping in free-recall learning," by John A. Robinson. Psychonomic Science, 1970, 21, 225. ROBINSON, J. A. Initial-recall grouping in frec-recal: learning. Psychonomic Science, 1970. 20.67-68. 